\title{
CULTIVOS HERBÁCEOS, VIÑEDOS Y OLIVARES. EL MANEJO TRADICIONAL DEL SUELO Y SUS CONSECUENCIAS EN LA EROSIÓN HÍDRICA
}

\author{
R. BIENES ${ }^{(1,2)^{*}}$, M. J. MARQUÉS ${ }^{(3)}$, \\ M. RUÍZ-COLMENERO ${ }^{(1)}$ \\ ${ }^{1}$ Instituto Madrileño de Investigación y Desarrollo Rural, Agrario y Alimentario \\ (IMIDRA). Apdo. correos 127. 28800-Alcalá de Henares (Madrid), Spain. \\ ${ }^{2}$ Departamento de Geología. Universidad de Alcalá. 28871-Alcalá de Henares (Madrid), Spain. \\ ${ }^{3}$ Departamento de Geología y Geoquímica. Universidad Autónoma de Madrid. \\ Campus de Cantoblanco, 28049 Tres Cantos (Madrid), Spain.
}

RESUMEN. La pérdida de productividad y el habitual uso de fertilizantes y pesticidas en los campos agrícolas españoles, nos hace reflexionar sobre la adecuación de los manejos utilizados. La erosión es uno de los procesos implicados en esta pérdida de productividad, por lo que se hace necesaria la evaluación de la gravedad de la misma. Esto puede servir en la toma de decisiones por parte de la sociedad, consumidores y/o agricultores, y para orientar a las autoridades competentes en la implementación de medidas. Así, durante 16 años hemos estudiado las pérdidas de suelo en los cultivos predominantes en España (cereal, olivar y viñedo) utilizando distintas técnicas. De este modo puede conocerse la repercusión de distintos manejos del suelo en nuestros terrenos agrícolas y saber si la tasa erosiva es o no tolerable según los criterios actuales. Se ha comprobado en diferentes escenarios que manejos con suelo desnudo como son el laboreo y/o el empleo de herbicidas, comprometen la conservación de los suelos, con tasas erosivas medias de hasta 20 t ha $\mathrm{a}^{-1}$ año $\mathrm{I}^{-1}$. Estas tasas van desde $1.3 \mathrm{tha}^{-1}$ año-1 en condiciones de baja erosividad, a las $93.5 \mathrm{t} \mathrm{ha^{-1 }}$ que se pueden producir en un solo evento como una tormenta de alta erosividad. En el caso de cultivo leñosos (olivar y viñedo), entre las alternativas estudiadas a estos manejos, se encuentra la vegetación espontánea que redujo la erosión a tasas de $1.76 \mathrm{tha}^{-1}$ año $\mathrm{n}^{-1}$, un valor aún al límite de lo admisible para los suelos estudiados. El manejo más eficaz en el control de la erosión fue el empleo de cubiertas de gramíneas permanentes y cubiertas sembradas con siega, con pérdidas de 0.24 y 0.32 tha $a^{-1}$ año $o^{-1}$ respectivamente. Así, se recomienda la utilización de cubiertas vegetales, que variarán en la especie elegida y su manejo según las características de la zona. 


\title{
Herbaceous crops, vineyards and olive groves. The traditional land management
} and its impact on water erosion

\begin{abstract}
The loss of productivity and the frequent use of fertilizers and pesticides in Spanish agricultural fields makes us reflect on the adequacy of the soil management used in our land. Soil erosion is one of the processes involved in this loss of productivity, showing the need/importance to evaluate its severity. Such an evaluation is essential for decision-making by society, consumers and/or farmers, and to guide the competent authorities to implement suitable measures. During the last 16 years we have been studying soil loss in the main crops in Spain (cereal, olive groves and vineyards) using different techniques. So, we can know the impact of different land management in our agricultural lands and whether the erosion rate is tolerable or not by today's standards. It has been tested in different settings that the managements with bare soil, such as tilling and/or the use of herbicides, threatens the soil conservation with average erosion rates up to $20 t \mathrm{ha}^{-1} \mathrm{yr}^{-1}$. These erosion rates vary from $1.3 \mathrm{tha}^{-1} \mathrm{yr}^{-1}$ with rains of low erosivity, to $93.5 \mathrm{th} \mathrm{a}^{-1}$ that can be produced in a single storm event highly erosive. In the case of woody crops (olives and grapes), we have studied different soil managements. The spontaneous vegetation reduced vineyard erosion rates to $1.76 \mathrm{tha}^{-1} \mathrm{yr}^{-1}$, value limit of what is considered admissible in our circumstances. The soil managements more effective to control erosion were permanent grass cover and mown cereal cover, with soil losses of 0.24 and $0.32 t h a^{-1} y r^{-1}$ respectively. Thus, we recommend the use of groundcovers, the management of which will vary depending on the characteristics of the area.
\end{abstract}

Palabras clave: erosión en campos agrícolas, cereal, olivar, viñedo, control de la erosión. Key words: erosion in agricultural fields, cereal, olive groves, vineyards, erosion control.

* Correspondencia: Instituto Madrileño de Investigación y Desarrollo Rural, Agrario y Alimentario (IMIDRA). Apdo. correos 127. 28800-Alcalá de Henares (Madrid). E-mail: ramon.bienes@madrid.org

\section{Introducción}

Más de un $99 \%$ de las calorías ingeridas por los seres humanos provienen de alimentos de la tierra (Pimentel, 2006) y según datos de la FAO (2005) un 42\% de la población depende de la actividad agrícola, junto a la caza, pesca y silvicultura. Es por esto que gran parte de nuestro suelo está dedicado a la agricultura: en Europa, los campos labrados y cultivos permanentes ocupan un $25 \%$ del suelo (EEA-ETC/LUSI, 2010). La agricultura tiene por tanto un peso importante en el medio ambiente, lo que supone, entre otras cosas, una responsabilidad con la disponibilidad del agua y la sostenibilidad del suelo que ocupa. 
Es por ello que un manejo inadecuado o el fomento de medidas irrespetuosas con el medio ambiente pueden afectar a una gran proporción de terreno y personas. Por ejemplo, la búsqueda de la maximización de la producción con incentivos erróneos, llevó durante muchos años en Europa a una intensificación de los cultivos con, entre otras consecuencias, un aumento de la erosión y la escorrentía (Boardman et al. 2003; Souchère et al., 2003), y en ocasiones a una sobreproducción innecesaria en perjuicio del propio agricultor. A pesar de esto, actualmente hay una creciente concienciación por la conservación del suelo. Esta concienciación apoya el desarrollo de proyectos científicos, que son los que proporcionan el conocimiento necesario para mejorar las medidas políticas relativas a una agricultura en consonancia con la conservación del suelo como la EU Soil Thematic Strategy (EC 2006) o la Council Regulations (EC) No 1257/1999 (Bazzofi, 2009). Esto se ve reflejado en acciones como la condicionalidad de las ayudas creada en el marco de la Política Agraria Comunitaria (Real Decreto 2352/2004). Pero el esfuerzo aún no ha llegado a cobrar la magnitud necesaria para frenar los procesos erosivos. Y es que parece que los agricultores europeos son conscientes de los problemas ambientales (Simoncini, 2009), aunque no del alcance de sus consecuencias, tal vez debido al largo plazo en el que ocurren. Por otro lado necesitan el apoyo de la sociedad para asumir el coste de la protección del suelo (Simoncini, 2009; Marques et al., 2010) para lo que será necesario un esfuerzo por educar y concienciar a la población (Calatrava et al., 2011).

En España, de los 16.2 Mha de tierras labradas, los cultivos herbáceos suponen el $73.2 \%$, el viñedo el $6.4 \%$ y el olivar el $13.8 \%$ (fuente, Instituto Nacional de Estadística, Anuario 2009). Se trata de una porción importante del territorio que se suele someter a manejos que dejan el suelo desnudo como el excesivo laboreo, la aplicación de herbicidas, etc. Al manejo perjudicial para el suelo, se suma que estos cultivos suelen encontrarse en pendiente (Romero, 1998).

La Política Agraria de la UE y el fortalecimiento de los mercados nacional e internacional, han fomentado la expansión de olivares y viñedos en tierras marginales, con pendientes pronunciadas (García-Ruiz, 2010). En estos cultivos las calles suelen estar desnudas gran parte del año, quedando el suelo expuesto a las precipitaciones, lo que lo hace vulnerable a la erosión hídrica de un modo directo, por impacto de la gota de lluvia, e indirecto por la escorrentía que se concentra en regueros, no escasamente presentes en nuestro paisaje agrícola. En estas circunstancias cobra especial importancia el régimen pluviométrico de tipo mediterráneo, con precipitaciones breves e intensas que son muy erosivas y pueden dar lugar a episodios extremos de pérdidas de suelo. De este modo las zonas agrícolas en España suelen aglutinar unas condiciones no muy propicias de al menos tres factores que son determinantes en la susceptibilidad de un suelo a ser erosionado: i) un clima con una pluviometría baja pero en forma de tormentas; ii) un manejo no siempre sostenible que rompe la estructura del suelo con excesivos laboreos y/o deja el suelo desnudo gran parte del año; y iii) la localización habitual de cultivos en zonas en pendiente.

Así, un manejo del suelo no siempre adecuado para las condiciones del lugar está produciendo altas tasas erosivas tanto en cereal como olivar y viñedo en España (García-Ruiz, 2010), y esta disminución en la profundidad de los suelos puede afectar a la 
producción de los cultivos (Ramos, 2006). Pero ¿hasta qué punto es grave el problema de la erosión en nuestros campos agrícolas?

Las consecuencias relacionadas con los procesos erosivos están llevando al abandono de los suelos agrícolas de toda Europa, abandono que ya afecta a miles de kilómetros cuadrados. Cuando se abandona un cultivo suele ser en las zonas ya altamente degradadas, lo que favorece aún más la erosión (Romero-Díaz, 2003). En las zonas mediterráneas la gravedad de este abandono radica en que los procesos de colonización de plantas son lentos (Garcia-Ruiz y Lana-Renault, 2011) y en la lenta tasa de formación del suelo en este clima (López-Bermúdez, 2002).

Creemos que es necesario evaluar a tiempo las consecuencias de los manejos en los procesos erosivos, y estudiar la idoneidad de alternativas. El estudio experimental de la erosión nos ofrece evidencias claras de la problemática y datos concretos al respecto, que pueden servir para cuantificar los costes medioambientales que incluyen el impacto social. Si se ofrecen datos concretos sobre la tolerancia de las tasas erosivas se facilitará la labor de las políticas tanto nacionales como europeas en materia de agricultura y suelos que pueden tener una base científica para la implementación de medidas adecuadas (Renschler y Harbor, 2002). Y así facilitará la concienciación de consumidores y agricultores, ya que sus decisiones están fuertemente influenciadas por factores socioeconómicos (Boardman et al., 2003). Esto puede facilitar que se frene la pérdida de productividad de los suelos y el aumento de superficie degradada, adaptándose dichas medidas a las condiciones regionales de cada área (Calatrava et al., 2011).

Así, desde 1994 se vienen estudiando las tasas erosivas en estos cultivos predominantes en España (cereal, olivar y viñedo) utilizando distintas técnicas. En este trabajo se hace una revisión de algunas de ellas. El periodo de estudio abarca 16 años, en los cuales se han estudiado las pérdidas de suelo y escorrentías generadas en parcelas sembradas con distintos cereales, en viñedos y olivares. El objetivo de este trabajo es realizar una revisión de diferentes pérdidas de suelo bajo distintas circunstancias para conocer la incidencia que están teniendo estos manejos del suelo en nuestros terrenos agrícolas y establecer si las pérdidas obtenidas son o no tolerables en función de los criterios actuales.

\section{Materiales y métodos}

\subsection{Zona de estudio}

Las localidades de estudio se encuentran en la zona centro peninsular, todas ellas bajo un clima mediterráneo semiárido, y tienen en común el uso secular del suelo para cultivos agrícolas. La temperatura media anual oscila entre los $12.6^{\circ} \mathrm{C}$ de Albaladejito (Cuenca) y los $14^{\circ} \mathrm{C}$ de Aranjuez (Madrid) y la pluviometría entre los $507 \mathrm{~mm}$ de Albaladejito y los $390 \mathrm{~mm}$ de Aranjuez (datos de la Agencia Estatal de Meteorología), presentando el resto de las localidades valores intermedios para estas dos variables. La vegetación natural, que podría alcanzar un climax dominado por Quercus coccifera y Pinus halepensis, está prácticamente desaparecida, a excepción de Albaladejito donde podemos encontrar una amplia 
superficie de pinar con una orla exterior de encinar y quejigar. Los representantes más abundantes de la vegetación son actualmente arbustos, pequeños ejemplares de Quercus, y retamas cuando los suelos son calcícolas, y tomillos (Thymus zygis) o espartos cuando los suelos son yesíferos (tabla 1). El paisaje dominante está constituido por extensiones de cultivos dedicados a cereales, olivos, almendros y viñedos.

Tabla 1. Precipitaciones máximas en 24 h. para los períodos de retorno de 2, 5 y 10 años (X2, X5 y X10) en las diferentes zonas de estudio (Elías Castillo y Ruiz Beltrán, 1979).

\begin{tabular}{|l|l|c|c|c|}
\hline Localidad & Observatorio & X2 (mm) & X5 (mm) & X10 (mm) \\
\hline Albaladejito & Cuenca & 53.6 & 70.9 & 82.5 \\
\hline Aranjuez & Aranjuez (Colegio) & 31.4 & 40.3 & 46.2 \\
\hline Marchamalo & Zorita de los Canes & 34.5 & 46.3 & 54.3 \\
\hline Encín & Alcalá-La Canaleja & 39.1 & 51.0 & 59.0 \\
\hline Campo Real & Alcalá-La Canaleja & 39.1 & 51.0 & 59.0 \\
\hline Villaconejos & Aranjuez & 31.4 & 40.3 & 46.2 \\
\hline Belmonte de Tajo & Villarejo de Salvanés & 35.0 & 50.6 & 61.1 \\
\hline
\end{tabular}

Las localidades en las que se han llevado a cabo los estudios cuyas conclusiones se exponen aquí, presentan lluvias máximas en 24 horas que pueden considerarse de medianamente erosivas a muy erosivas. En la tabla 1 figuran estas precipitaciones máximas en 24 h. para diferentes periodos de recurrencia.

Las características edáficas principales de los suelos estudiados, junto con su clasificación, las encontramos en la tabla 2.

Tabla 2. Localidades de estudio, ubicación y características del suelo.

\begin{tabular}{|c|c|c|c|c|c|c|c|}
\hline Localidades & $\begin{array}{c}\text { Alcalá } \\
\text { de Henares }\end{array}$ & Aranjuez & Marchamalo & Albaladejito & $\begin{array}{c}\text { Belmonte de } \\
\text { Tajo }\end{array}$ & Campo Real & Villaconejos \\
\hline $\begin{array}{l}\text { Huso } \\
\text { Coordenadas } \\
\text { UTM }\end{array}$ & $\begin{array}{l}30 \mathrm{~T} \\
\mathrm{X}: 473355 \\
Y: 4485991\end{array}$ & $\begin{array}{l}30 \mathrm{~T} \\
\mathrm{X}: 455219 \\
Y: 4436371\end{array}$ & $\begin{array}{l}30 \mathrm{~T} \\
\mathrm{X}: 481845 \\
\mathrm{Y}: 4503510\end{array}$ & $\begin{array}{l}30 \mathrm{~T} \\
\mathrm{X}: 568167 \\
Y: 4434684\end{array}$ & $\begin{array}{l}30 \mathrm{~T} \\
\mathrm{X}: 475919 \\
\mathrm{Y}: 4440274\end{array}$ & $\begin{array}{l}30 \mathrm{~T} \\
\mathrm{X}: 468169 \\
\mathrm{Y}: 4467525\end{array}$ & $\begin{array}{l}30 \mathrm{~T} \\
\mathrm{X}: 452424 \\
Y: 4436608\end{array}$ \\
\hline Pendiente & $6 \%$ & $10 \%$ & $12.3 \%$ & $8.9 \%$ & $12 \%$ & $14 \%$ & $8 \%$ \\
\hline Pedregosidad & $17 \%$ & inapreciable & $25 \%$ & $<1 \%$ & inapreciable & $60 \%$ & inapreciable \\
\hline Textura & $\begin{array}{l}\text { Franco } \\
\text { arcillosa }\end{array}$ & Arcillosa & Arcillosa & $\begin{array}{l}\text { Franco } \\
\text { arenosa }\end{array}$ & $\begin{array}{l}\text { Franco } \\
\text { arcillosa }\end{array}$ & $\begin{array}{l}\text { Franco } \\
\text { arcillosa }\end{array}$ & $\begin{array}{l}\text { Franco } \\
\text { arenosa }\end{array}$ \\
\hline $\mathrm{pH}_{(\mathrm{H} 2 \mathrm{O})} 1 / 2.5$ & 8.2 & 7.4 & 7.5 & 7.4 & 7.9 & 8.7 & 8.5 \\
\hline $\begin{array}{l}\text { Materia } \\
\text { orgánica }\end{array}$ & $1.1 \%$ & $1.8 \%$ & $1.1 \%$ & $0.6 \%$ & $0.85 \%$ & $1.27 \%$ & $0.38 \%$ \\
\hline $\begin{array}{l}\text { Clasificación } \\
\text { del suelo } \\
\text { (S. Taxonomy) }\end{array}$ & $\begin{array}{c}\text { Typic } \\
\text { Calcixerepts }\end{array}$ & $\begin{array}{c}\text { Cambic } \\
\text { Gipsiorthid }\end{array}$ & $\begin{array}{c}\text { Typic } \\
\text { Rhodoxeralf }\end{array}$ & $\begin{array}{c}\text { Calcic } \\
\text { Haploxeralf }\end{array}$ & $\begin{array}{c}\text { Typic } \\
\text { Calcixerepts }\end{array}$ & $\begin{array}{c}\text { Typic } \\
\text { Calcixerepts }\end{array}$ & $\begin{array}{c}\text { Calcic } \\
\text { Haploxeralf }\end{array}$ \\
\hline
\end{tabular}




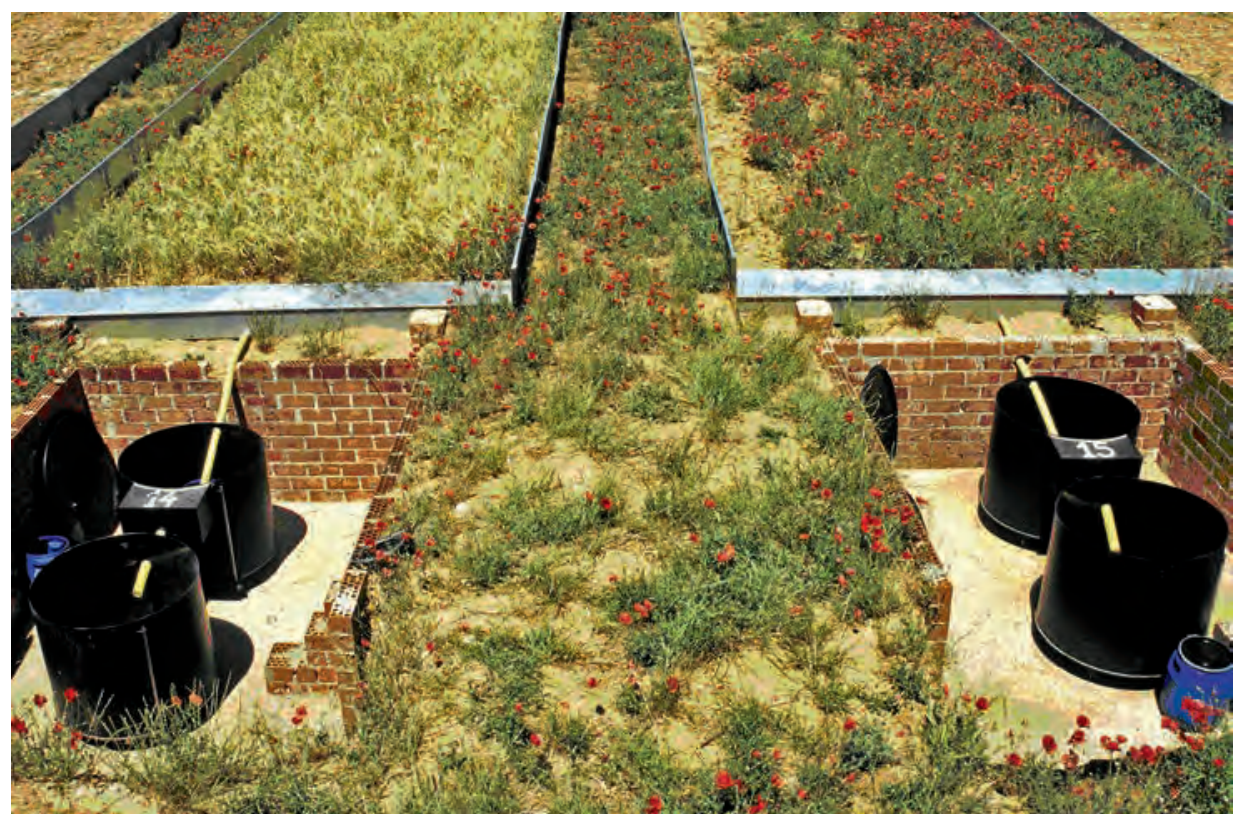

Figura 1. Parcelas de erosión de $80 \mathrm{~m}^{2}(4 \mathrm{~m} \times 20 \mathrm{~m})$, en la cota inferior están dotadas de un canalón de recogida de sedimentos que se conecta con dos depósitos de 500 litros; el segundo depósito recoge 1/5 del volumen que pudiera rebosar del primero. La parcela de la izquierda presenta un cultivo de cebada, la de la derecha tiene vegetación espontánea.

\subsection{Erosión}

A lo largo de los años se han empleado distintas metodologías para estimar la erosión hídrica analizando los resultados bajo lluvias naturales y también bajo lluvias simuladas.

Parcelas de erosión. El procedimiento consiste en acotar una superficie del suelo y medir procesos de infiltración y escorrentía en dichas superficies. Se han empleado diferentes dimensiones de parcelas, todas ellas estuvieron delimitadas por chapas metálicas enterradas que tenían en su base un colector para recoger los sedimentos erosionados y un sistema de recogida de aguas de escorrentía.

Se han utilizado desde parcelas de $80 \mathrm{~m}^{2}$ (4 x $20 \mathrm{~m}$; fig. 1), estas parcelas son similares a las utilizadas para desarrollar el modelo USLE (Wischmeier y Smith, 1978), hasta parcelas de $1 \mathrm{~m}^{2}(0.5$ x $2 \mathrm{~m}$; fig. 2$)$ y de $2 \mathrm{~m}^{2}$ (0.5 x $4 \mathrm{~m}$; fig. 7$)$.

Lluvia simulada. En el clima de las zonas de estudio es muy difícil predecir el régimen de lluvias. A esto hay que añadir la distribución irregular de los eventos extremos que son los más erosivos. Para evitar estos inconvenientes se puede usar la lluvia simulada, que además es un método que puede ser controlado a voluntad por los investigadores en cuanto a duración e intensidad, lo que hace de esta metodología un instrumento 


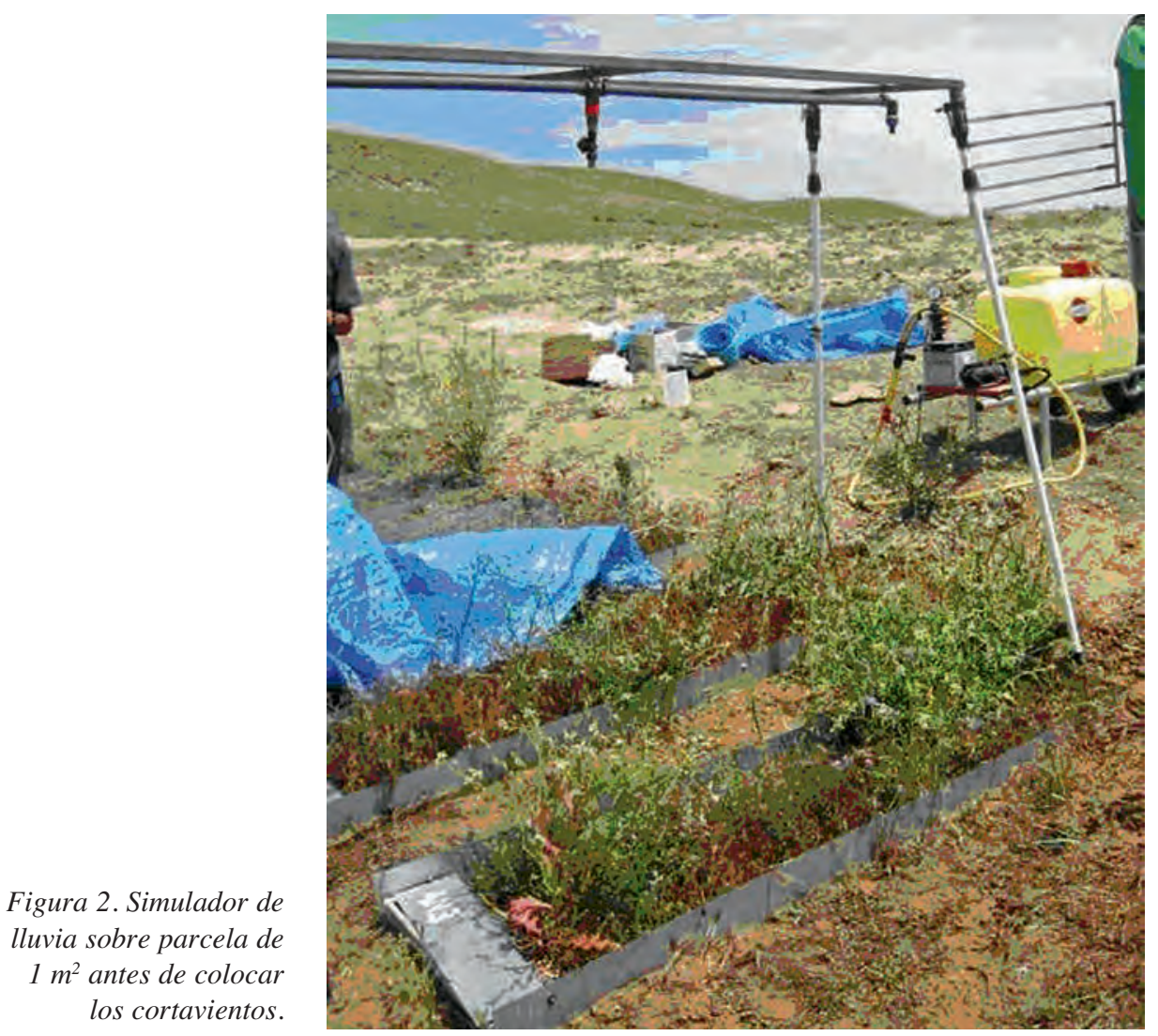

muy útil para el estudio de la erosión. A pesar de que este tipo de lluvias no puede ser comparado plenamente con lluvias naturales, sí puede aportar información útil en la comparación de tratamientos, tipos de suelo, estados de degradación, etc. Además, soluciona el problema de la escasez de eventos lluviosos que podrían retrasar la obtención de datos (Cerdá, 1999) en ambientes mediterráneos, donde unos pocos eventos pueden producir la mayor parte de la pérdida de suelo (González-Hidalgo et al., 2005; Bienes y Marqués, 2007). Se han utilizado simuladores diseñados para realizar lluvias sobre todas las parcelas de erosión consideradas, cuyas superficies son de 1,2 y $80 \mathrm{~m}^{2}$.

En todas las parcelas se realizaron pruebas de calibración de los simuladores para conseguir las intensidades elegidas y para verificar un coeficiente de uniformidad (CU) admisible (Christiansen, 1942). Se utilizaron boquillas de Spraying systems Inc. 3/8 HHMFP 11514 para los simuladores de 1 y $80 \mathrm{~m}^{2}$ y boquillas $1 / 3 \mathrm{HH} 35 \mathrm{~W}$ para el simulador de $2 \mathrm{~m}^{2}$.

La intensidad y duración de las tormentas simuladas fueron elegidas de acuerdo con un compromiso entre la intensidad y la frecuencia de las tormentas en la zona. Trabajos 
realizados en Europa han considerado como energías cinéticas mínimas valores de 12.8 $\mathrm{J} \mathrm{m}^{-2} \mathrm{~mm}^{-1}$ (Zanchi y Torri, 1980) o de $15.8 \mathrm{~J} \mathrm{~m}^{-2} \mathrm{~mm}^{-1}$ (Coutinho yThomas, 1995). La calibración de los simuladores arrojó valores de energía cinética de 13.5 a $18 \mathrm{~J} \mathrm{~m}^{-2} \mathrm{~mm}^{-1}$, que son tormentas moderadas que pueden tener una frecuencia anual. A continuación se describen más en detalle las características de los simuladores empleados.

- Simulador de $1 \mathrm{~m}^{2}$. Se trata de un simulador desmontable, que puede utilizarse en cualquier lugar, siempre que la pendiente no exceda del 15\%. Está formado por un marco de 0.5 x $2 \mathrm{~m}^{2}$, mantenido sobre 4 tubos telescópicos de $2 \mathrm{~m}$. El marco tiene una tubería de PVC con una boquilla 3/8 HHMFP 115 14. Esta tubería está alimentada desde un depósito provisto con una bomba que funciona mediante una batería de 12 v (fig. 2).

La boquilla tiene un ángulo de apertura grande, de $112^{\circ}$, lo que permite hacer una lluvia sobre dos parcelas adyacentes con un CU del $82 \%$, cuando se emplea una presión de $1.5 \pm 0.2 \mathrm{~kg} \mathrm{~cm}^{-2}$. En estas condiciones, la intensidad de la lluvia es de $27.8 \mathrm{~mm} \mathrm{~h}^{-1}$. El diámetro D50 de la gota de lluvia es $1.04 \mathrm{~mm}$. La energía cinética es de $18 \mathrm{~J} \mathrm{~m}^{-2} \mathrm{~mm}^{-1}$ (Marqués et al., 2005).

- Simulador de lluvia de $2 \mathrm{~m}^{2}$. Este simulador tiene un diseño similar al de $1 \mathrm{~m}^{2}$, aunque el bastidor y los soportes son necesariamente más robustos y pesados, también es portátil y puede desmontarse parcialmente y se utilizó sobre parcelas que tienen $4 \mathrm{~m}$ de longitud y $0.5 \mathrm{~m}$ de anchura. Dispone de una única tubería central con 2 boquillas Spraying Systems 1/3 HH 35 W de cono lleno separadas entre sí $1.5 \mathrm{~m}$ y que abarcan una superficie $4 \mathrm{~m}^{2}$ de suelo con un coeficiente de CU de $86 \%$. El diámetro D50 de las gotas fue de $1.85 \mathrm{~mm}$. La intensidad de las lluvias fue de 2.16 $\mathrm{mm} \mathrm{min}^{-1}$ a una presión de $1.5 \pm 0.2 \mathrm{Kg} \mathrm{cm}^{-2}$. Dado que las lluvias se realizaron durante 15 minutos, resultó una energía cinética equivalente de $17.83 \mathrm{~J} \mathrm{~m}^{-2} \mathrm{~mm}^{-1}$ (Ruiz-Colmenero et al., 2010; Martínez-Mena et al., 2001).

- Simulador de lluvia de $80 \mathrm{~m}^{2}$. Es un simulador fijo, pero puede desplazarse sobre las 9 parcelas de $80 \mathrm{~m}^{2}$ que están situadas en la localidad de Aranjuez. Dos tuberías de 20 m conectadas entre sí en un circuito cerrado, están suspendidas de cables de acero entre dos hileras de pilares metálicos que se alinean en la cabecera y en la base de las parcelas USLE. El sistema tiene 20 boquillas, con una separación entre boquillas de $2 \mathrm{~m}$ de distancia. Con este simulador se produce una lluvia de $20.8 \mathrm{~mm}$ $\mathrm{h}^{-1}$ a una presión de $1.3 \pm 0.2 \mathrm{~kg} \mathrm{~cm}^{-2}$. El coeficiente de uniformidad de la lluvia es del $80 \%$ y el diámetro D50 de las gotas es de $1.6 \mathrm{~mm}$. La energía cinética resultante es de $13.5 \mathrm{~J} \mathrm{~m}^{-2} \mathrm{~mm}^{-1}$ (Marqués et al., 2007) (fig. 3).

Lluvias naturales bajo eventos erosivos. Erosión en regueros y entre regueros. El régimen de lluvias del clima mediterráneo semiárido de la zona de estudio tiene como característica la existencia de tormentas aisladas, de frecuencia irregular que son el desencadenante principal de la pérdida de suelo desde el punto de vista cuantitativo. Cuando se produce este tipo de tormentas sobre suelos labrados en pendiente, como los que son habituales en los olivares, se produce una red de drenaje estructurada en regue- 

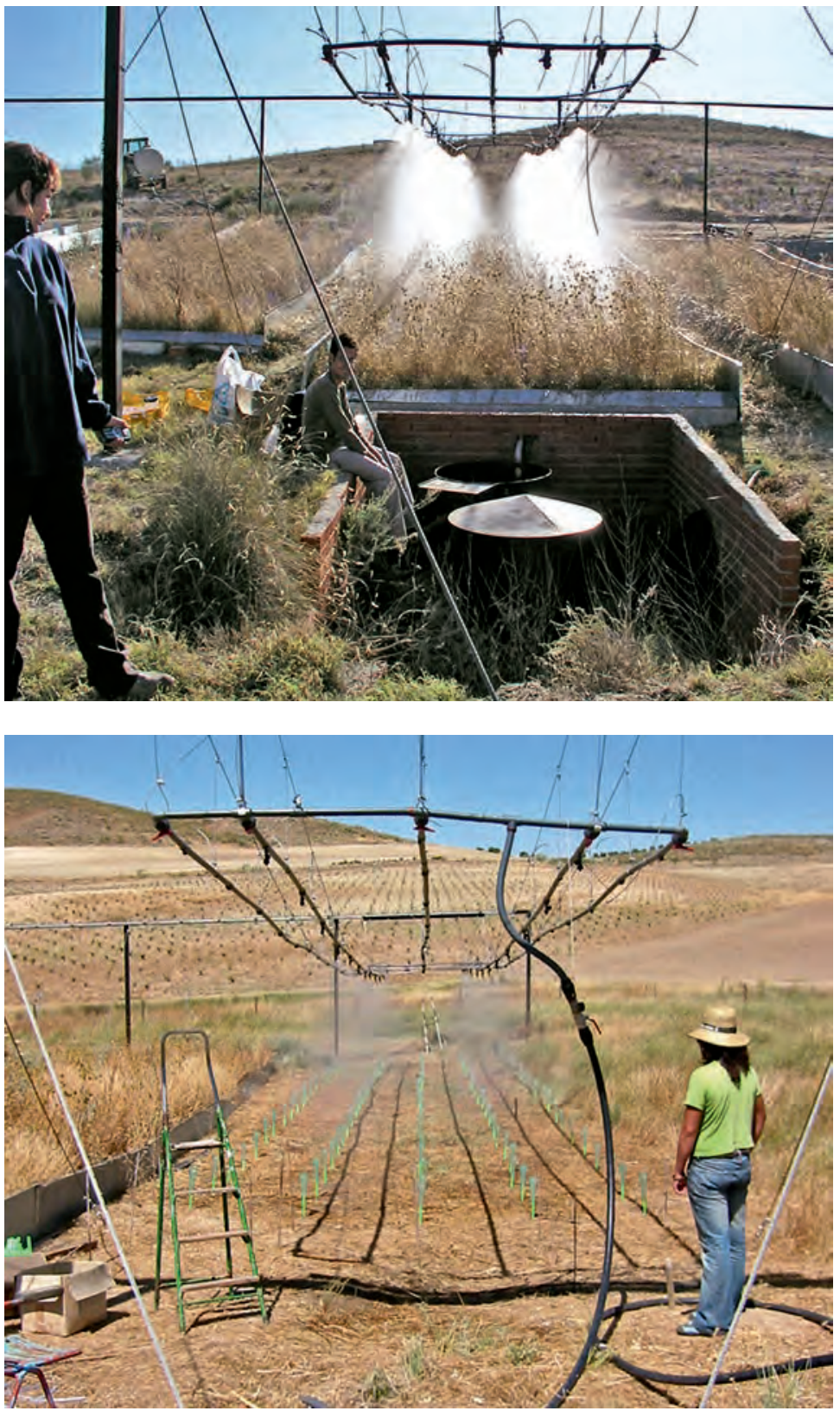

Figura 3. Simulador de lluvia sobre parcela de $80 \mathrm{~m}^{2}$ con vegetación espontánea, en la base se aprecian los depósitos de 500 litros para la recogida de la escorrentía. El simulador consta de 2 tuberías conectadas con 10 boquillas en cada tubería, separadas por 2 metros. Abajo, ensayos de calibración con pluviómetros para valoración de intensidad, pluviometría y coeficiente de homogeneidad. Al fondo se puede apreciar el paisaje dominante en esta zona de Aranjuez. 
ros interconectados que dan lugar a mayores arrastres de suelo. En este trabajo se mide la erosión producida en una pequeña cuenca de $200 \mathrm{~m}^{2}$. La anchura y profundidad media de los regueros formados se midió cada dos metros. La erosión entre regueros se midió usando 15 parcelas de $1 \mathrm{~m}^{2}$ situadas en la zona (Bienes y Marqués, 2008).

\section{Resultados y Discusión}

\subsection{Cubiertas herbáceas de gramíneas (cebada, Hordeum vulgare); vegetación espontánea y suelo desnudo empleando herbicidas}

La cubierta de cebada representa uno de los cultivos con mayor extensión en España, según los datos del Instituto Nacional de Estadística a este cereal se dedica más de la mitad $(50.7 \%)$ del terreno labrado destinado a cereales, lo que supone actualmente unos 3.4 Mha. En este trabajo se estudia la tasa anual de pérdida de suelo de este cultivo manejado bajo mínimo laboreo, en comparación con otros dos usos del suelo: vegetación espontánea, pasto mejorado mediante la introducción de leguminosas adaptadas a cada localidad (Astragalus cicer en Albaladejito, Medicago sativa en Alcalá de Henares, Hedysarum spinosissimum y Hedysarum humile en Aranjuez); y suelo desnudo mediante la aplicación de herbicidas. Las diferencias en las pérdidas de suelo apoyan las recomendaciones de la actual Política Agraria Comunitaria, que impone la utilización de medidas agroambientales orientadas a la protección del suelo (condicionalidad de las ayudas), en especial en los periodos de barbecho mediante el empleo de cualquier tipo de cubierta vegetal viva o muerta. También pueden aportar datos para conocer la evolución de tierras abandonadas por falta de productividad en las que se permite el crecimiento espontáneo de la vegetación.

En estos estudios se emplearon herbicidas sistémicos como el glifosato y también herbicidas de pre-emergencia y post-emergencia como el oxadiazón para mantener el suelo desnudo. El mínimo laboreo aplicado al cultivo de cebada se reducía a un pase de chisel antes de la siembra, realizado imitando el uso tradicional, es decir, con los surcos del la labor a favor de la pendiente. Tras la cosecha del cereal, se dejaron los restos vegetales sobre el suelo hasta el momento del siguiente laboreo y siembra. La vegetación espontánea se dejó evolucionar sin intervención. En la figura 1 se pueden observar estos dos últimos manejos.

Los ensayos se repitieron en cuatro localidades: Aranjuez (Madrid), Alcalá de Henares (Madrid), Marchamalo (Guadalajara) y Albaladejito (Cuenca) (tabla 2). Se tuvieron en cuenta los eventos que superaron los $10 \mathrm{~mm}$ de pluviometría.

En la tabla 3 se observa que en el periodo de estudio, de 1994 a 1997, las pérdidas de suelo son varios órdenes de magnitud mayores en el suelo desnudo que en cualquiera de las dos cubiertas, en especial en la localidad de Marchamalo. La mayor parte de la pérdida de suelo se produce en unos pocos eventos (Bienes y Torcal, 1997). La cebada se desarrolla formando macollas que aglutinan eficazmente el suelo, previniendo su pérdida, tanto durante su crecimiento como tras la cosecha, cuya rastrojera se mantiene hasta el final del otoño. 
Tabla 3. Pérdida de suelo y coeficiente de escorrentía (escorrentía/pluviometría) en los distintos usos: suelo desnudo con herbicidas (S.D.), cultivo de cebada, vegetación espontánea (V.E.) y pastizal. Parcelas tipo USLE de $80 \mathrm{~m}^{2}$.

\begin{tabular}{|c|c|c|c|c|c|c|c|c|c|}
\hline \multirow{2}{*}{$\begin{array}{l}\text { Localidad } \\
\text { y año }\end{array}$} & \multirow{2}{*}{$\begin{array}{l}\mathrm{N}^{0} \text { de } \\
\text { eventos }\end{array}$} & \multicolumn{4}{|c|}{$\begin{array}{l}\text { Pérdida de suelo } \\
\qquad\left(\mathrm{t} \mathrm{ha}^{-1} \mathrm{año}^{-1}\right)\end{array}$} & \multicolumn{4}{|c|}{$\begin{array}{c}\text { Coeficiente de escorrentía } \\
(\%)\end{array}$} \\
\hline & & S.D. & Cebada & V.E. & Pastizal & S.D. & Cebada & V.E. & Pastizal \\
\hline \multicolumn{10}{|l|}{ Aranjuez } \\
\hline 1994 & 8 & 1.92 & 0.023 & 0.487 & 0.391 & 6.72 & 0.32 & 3.52 & 4.82 \\
\hline 1995 & 7 & 19.50 & 0.276 & 1.775 & 8.868 & 9.35 & 0.94 & 1.61 & 8.49 \\
\hline 1996 & 11 & 14.13 & 0.957 & 0.064 & 0.715 & 13.92 & 2.65 & 0.94 & 3.34 \\
\hline 1997 & 14 & 5.95 & 2.467 & 0.007 & 0.007 & 13.09 & 1.50 & 0.98 & 0.78 \\
\hline \multicolumn{10}{|l|}{ Alcalá de H. } \\
\hline 1994 & 15 & 6.95 & 0.008 & 0.154 & 3.223 & 4.93 & 0.38 & 1.12 & 3.39 \\
\hline 1995 & 10 & 4.41 & 0.122 & 0.038 & 0.076 & 6.60 & 0.21 & 0.70 & 0.54 \\
\hline 1996 & 10 & 2.55 & 0.088 & 0.001 & 0.020 & 10.29 & 0.45 & 0.90 & 0.81 \\
\hline 1997 & 17 & 13.75 & 0.230 & 0.019 & 0.012 & 11.25 & 0.98 & 0.81 & 0.76 \\
\hline \multicolumn{10}{|l|}{ Marchamalo } \\
\hline 1994 & 12 & 0.12 & 0.023 & 0.023 & 0.012 & 0.62 & 0.51 & 0.74 & 0.67 \\
\hline 1995 & 11 & 24.06 & 0.177 & 0.082 & 0.128 & 7.25 & 0.51 & 0.75 & 0.65 \\
\hline 1996 & 11 & 63.93 & 0.087 & 0.069 & 0.025 & 13.29 & 0.78 & 0.75 & 0.81 \\
\hline 1997 & 15 & 23.73 & 0.036 & 0.020 & 0.012 & 7.68 & 0.35 & 0.67 & 0.66 \\
\hline \multicolumn{10}{|l|}{ Albaladejito } \\
\hline 1994 & 7 & 0.04 & 0.020 & 0.032 & 0.024 & 0.73 & 0.16 & 0.43 & 0.59 \\
\hline 1995 & 6 & 1.26 & 0.030 & 0.025 & 0.095 & 1.98 & 0.18 & 0.24 & 0.75 \\
\hline 1996 & 7 & 2.13 & 0.056 & 0.048 & 0.016 & 4.74 & 0.38 & 0.75 & 0.75 \\
\hline 1997 & 12 & 4.91 & 0.055 & 0.002 & 0.001 & 6.58 & 0.54 & 0.39 & 0.87 \\
\hline
\end{tabular}

En la tabla 4 se observan los valores medios del periodo de estudio y se comprueba que la vegetación espontánea otorga una protección al suelo ligeramente superior que el cultivo de cebada bajo mínimo laboreo. En todo caso, esta mejor disposición se consigue a medida que transcurre el tiempo, pues en un primer momento, según se observa en los datos de la tabla 3, la vegetación espontánea (V.E.) tenía mayores pérdidas que el cultivo de cebada. Bienes et al. (2005) ya habían comprobado esta eficiencia de la vegetación espontánea a medio plazo. 
Tabla 4. Medias anuales de pérdida de suelo considerando todo el periodo de estudio, de 1994 a 1997. Dimensiones de la parcela: $80 \mathrm{~m}^{2}$.

\begin{tabular}{|l|c|c|c|c|}
\hline & \multicolumn{3}{|c|}{ Pérdida de suelo $\left(\mathbf{t ~ h a}^{-\mathbf{1}} \mathbf{a n ̃ o}^{-\mathbf{1}}\right)$} \\
\hline Localidad & Suelo desnudo & Cebada & V.E. & Pastizal \\
\hline Aranjuez & $11.85 \pm 9.01$ & $0.93 \pm 1.1$ & $0.58 \pm 0.82$ & $2.50 \pm 4.26$ \\
\hline Alcalá de H. & $6.92 \pm 4.90$ & $0.11 \pm 0.09$ & $0.07 \pm 0.07$ & $0.83 \pm 1.59$ \\
\hline Marchamalo & $27.96 \pm 26.47$ & $0.08 \pm 0.07$ & $0.05 \pm 0.03$ & $0.04 \pm 0.06$ \\
\hline Albaladejito & $2.09 \pm 2.07$ & $0.04 \pm 0.02$ & $0.03 \pm 0.02$ & $0.03 \pm 0.04$ \\
\hline
\end{tabular}

Las analíticas de estos sedimentos pusieron de manifiesto unas pérdidas de nutrientes importantes (Bienes y Marqués, 2002), cuyos valores se exponen en la tabla 5. Destaca la gran pérdida de materia orgánica que puede llegar a registrarse, cuando mantenemos el suelo desnudo.

Tabla 5. Pérdidas totales de nutrientes en los sedimentos en el periodo 1994-1997, expresadas en $\mathrm{kg} \cdot \mathrm{ha}^{-1}$.

\begin{tabular}{|l|c|c|c|c|c|c|}
\hline Localidad / Cubierta suelo & Ca & Mg & Na & K & $\begin{array}{c}\text { Materia } \\
\text { orgánica }\end{array}$ & $\begin{array}{c}\text { Fósforo } \\
\text { asimilable }\end{array}$ \\
\hline Alcalá de Henares (Madrid) & \multicolumn{7}{|l|}{} \\
\hline Desnudo & 79.9 & 4.3 & 5.5 & 7.4 & 503 & 0.99 \\
\hline Cebada & 1.0 & 0.1 & 0.1 & 0.2 & 9.8 & 0.03 \\
\hline Espontánea & 0.1 & 0.0 & 0.0 & 0.0 & 5.7 & 0.01 \\
\hline Pastizal & 0.4 & 0.1 & 0.1 & 0.1 & 75 & 0.01 \\
\hline Aranjuez (Madrid) & 127.8 & 7.2 & 9.8 & 13.6 & 1768 & 3.73 \\
\hline Desnudo & 6.3 & 0.3 & 0.2 & 0.5 & 220 & 0.35 \\
\hline Cebada & 5.1 & 0.3 & 0.5 & 0.8 & 106 & 0.16 \\
\hline Espontánea & 35.9 & 1.9 & 2.3 & 4.4 & 507 & 0.40 \\
\hline Pastizal & 518.0 & 27.4 & 32.6 & 45.3 & 1545 & 5.20 \\
\hline Marchamalo (Guadalajara) & 0.7 & 0.1 & 0.1 & 0.2 & 8.1 & 0.04 \\
\hline Desnudo & 0.6 & 0.0 & 0.1 & 0.1 & 5.0 & 0.01 \\
\hline Cebada & 0.5 & 0.0 & 0.1 & 0.1 & 4.4 & 0.01 \\
\hline Espontánea & 18.5 & 1.6 & 1.6 & 3.3 & 348 & 0.51 \\
\hline Pastizal & 0.3 & 0.1 & 0.04 & 0.1 & 3.3 & 0.02 \\
\hline Albadalejito (Cuenca) & 0.2 & 0.0 & 0.02 & 0.01 & 5.5 & 0.01 \\
\hline Desnudo & 0.2 & 0.0 & 0.03 & 0.07 & 4.6 & 0.01 \\
\hline Cebada & 5 & \\
\hline Espontánea & \multicolumn{7}{|l|}{} \\
\hline Pastizal & \multicolumn{7}{|l|}{} \\
\hline
\end{tabular}


Los coeficientes de escorrentía medios para el período 1994 a 1997 (tabla 6), arrojan valores muy similares tanto para el cultivo de cebada bajo mínimo laboreo, como para la vegetación espontánea y el pastizal, pero a diferencia de la pérdida de suelo, el cultivo es algo más eficaz para disminuir la escorrentía que las otras dos cubiertas vegetales, lo que atribuimos a la mayor homogeneidad que presenta la distribución de la vegetación en el caso del cultivo.

Tabla 6. Coeficientes de escorrentía medio anual considerando todo el periodo de estudio: de 1994 a 1997. Dimensiones de la parcela: $80 \mathrm{~m}^{2}$.

\begin{tabular}{|l|c|c|c|c|}
\hline Localidad & Suelo desnudo & Cebada & V.E. & Pastizal \\
\hline Aranjuez & $10.8 \pm 3.4$ & $1.4 \pm 1.0$ & $1.8 \pm 1.2$ & $4.4 \pm 3.2$ \\
\hline Alcalá de H. & $8.3 \pm 3.0$ & $0.5 \pm 0.3$ & $0.9 \pm 0.2$ & $1.4 \pm 1.3$ \\
\hline Marchamalo & $7.2 \pm 5.2$ & $0.5 \pm 0.2$ & $0.7 \pm 0.04$ & $0.7 \pm 0.1$ \\
\hline Albaladejito & $3.5 \pm 2.6$ & $0.3 \pm 0.2$ & $0.5 \pm 0.2$ & $0.7 \pm 0.1$ \\
\hline
\end{tabular}

En unos ensayos realizados posteriormente (2004), sólo en la localidad de Aranjuez, se comparó de nuevo la vegetación espontánea desarrollada (100\% cobertura vegetal) con el suelo prácticamente desnudo (cobertura vegetal 3.5\%). En este caso, se emplearon parcelas también de $80 \mathrm{~m}^{2}$, pero se utilizó simulación de lluvia para estudiar la tasa de erosión y el coeficiente de escorrentía minuto a minuto en una lluvia de $21 \mathrm{~mm}$ de pluviometría durante 15 minutos. Se trata de una lluvia moderada-fuerte cuyo período de recurrencia es anual (Elías Castillo y Ruiz Beltrán, 1979).

Las lluvias sobre parcelas con vegetación prácticamente no produjeron escorrentía, arrojando valores del coeficiente de escorrentía de $0.04 \%$. Sin embargo, el suelo desnudo arrojó un valor medio del coeficiente de escorrentía del 35\%. Esta gran diferencia se debe no sólo a la ausencia de cobertura vegetal, también a la presencias de una costra de splash que, en los primeros 3-4 milímetros del suelo presentaba una densidad aparente de $1.5 \mathrm{~g} \mathrm{~cm}^{-3}$ en contraste con la densidad de $1.25 \mathrm{~g} \mathrm{~cm}^{-3}$ del suelo subyacente. Los datos del coeficiente de escorrentía de esta misma región bajo lluvias naturales oscila entre el $4 \%$ (Bienes et al., 2001) y el 14\% (Nicolau et al., 2002).

En cuanto a la pérdida de suelo, en la tabla 7 se observa que la fracción arrastrada en suspensión por el agua de escorrentía es importante. Del total de $74 \mathrm{~kg} \mathrm{ha}^{-1}$ perdidos en esta lluvia, el $77 \%$ se pierde en suspensión, y el resto se moviliza por acarreo y queda retenido en el canal de recogida de sedimentos de la parcela. Dado que se trata de una lluvia de 27.8 $\mathrm{mm}$, esta pérdida podría asimilarse a $2.66 \mathrm{~kg} \mathrm{ha}^{-1} \mathrm{~mm}^{-1}$; puesto que la zona recibe anualmente $390 \mathrm{~mm}$ al año, si las diferentes lluvias a lo largo del año presentaran intensidades similares, la pérdida anual resultante sería $1.04 \mathrm{t} \mathrm{ha}^{-1}$. Este valor estimado, está en el límite inferior de las pérdidas encontradas por Nicolau et al. (2002) para lluvias naturales, y que oscila entre 1.3 y $28 \mathrm{t} \mathrm{ha}^{-1} \mathrm{año}^{-1}$. Haciendo esta misma abstracción, el suelo con vegetación espontánea perdió $0.3 \mathrm{~kg} \mathrm{ha}^{-1}$ en esta lluvia, es decir, $0.01 \mathrm{~kg} \mathrm{ha}^{-1} \mathrm{~mm}^{-1}$, lo que 
supondría anualmente $0.004 \mathrm{t} \mathrm{ha}^{-1} \mathrm{año}^{-1}$, valor que coincide con las pérdidas anuales calculadas para el periodo 1994-1997 para vegetación espontánea presentadas en la tabla 4.

Tabla 7. Pérdida de suelo bajo una lluvia simulada de $21 \mathrm{~mm}$ en parcelas de $80 \mathrm{~m}^{2}$.

\begin{tabular}{|l|c|c|c|c|}
\hline Manejo del suelo & $\mathbf{n}$ & $\begin{array}{c}\text { Erosión en } \\
\text { suspensión } \\
\left(\mathbf{k g ~ h a}^{-1}\right)\end{array}$ & $\begin{array}{c}\text { Erosión en } \\
\text { acarreo } \\
\left(\mathbf{k g ~ h a}^{-1}\right)\end{array}$ & $\begin{array}{c}\text { Erosión } \\
\text { total } \\
\left(\mathbf{k g ~ h a}^{-1}\right)\end{array}$ \\
\hline Suelo desnudo (cobertura 3.5\%) & 8 & $57 \pm 35$ & $17 \pm 15$ & $74 \pm 43$ \\
\hline V.E. (cobertura $\approx 100 \%)$ & 8 & inapreciable & $0.3 \pm 0.5$ & $0.3 \pm 0.5$ \\
\hline
\end{tabular}

\subsection{Erosión con diferentes manejos del suelo en girasol}

En ensayos realizados durante las campañas agrícolas 2002/03, 2003/04 y 2004/05, se han cuantificado las pérdidas de suelo bajo cultivo de girasol con diferentes manejos agronómicos en los que se combinaba la dirección del laboreo (por curvas de nivel o a favor de la máxima pendiente) y diferentes fechas de enterrado del rastrojo, tras la recolección (octubre) y tras las lluvias otoñales (diciembre). Es de sobra sabido que el uso y manejo que se da al suelo tiene una fuerte incidencia sobre las tasas erosivas y la generación de escorrentía (Dunjó et al., 2004; Arshad et al., 1997; Sturgul et al., 1990). Respecto a la influencia de la dirección del laboreo, estudios llevados a cabo con maíz demostraron que se podía reducir la erosión entre el 40 y 65\% (Basic et al., 2004). Otros autores han apuntado las ventajas que puede ofrecer la presencia de residuos en superficie con el fin de mitigar la eosión (Rainbault et al., 1990).

En este sentido hay que decir que en la zona de estudio se registran lluvias intensas y muy erosivas durante el mes de octubre, las cuales representan el $47 \%$ de la pérdida total de suelo, por lo que el comportamiento del suelo en este mes resulta clave.

Las tasas de erosión obtenidas figuran en la tabla 8. La dirección del laboreo se mostró como el factor más condicionante, con independencia del manejo del rastrojo. Tanto la dirección del laboreo como el manejo del rastrojo tuvieron una amplia repercusión sobre las tasas de erosión.

Tabla 8. Tasas medias de erosión y escorrentía registradas para el periodo estudiado.

\begin{tabular}{|l|c|c|}
\hline Cobertura vegetal & $\begin{array}{c}\text { Tasa de } \\
\text { erosión }\left(\mathbf{k g ~ h a}^{-\mathbf{1}}\right)\end{array}$ & $\begin{array}{c}\text { Escorrentía } \\
(\mathbf{m m})\end{array}$ \\
\hline Labor a favor de la pendiente y enterrado tardío rastrojo (diciembre) & 2983.7 & 13.0 \\
\hline Labor a favor de la pendiente y enterrado temprano rastrojo (octubre) & 1053.8 & 6.5 \\
\hline Labor por curvas de nivel dejando y enterrado tardío rastrojo (diciembre) & 1293.5 & 9.1 \\
\hline Labor por curvas de nivel y enterrado temprano rastrojo (octubre) & 662.8 & 6.7 \\
\hline
\end{tabular}


A igualdad de la dirección del laboreo, las pérdidas de suelo que se obtuvieron cuando se realizó un enterrado temprano del rastrojo fueron un 34 y $51 \%$ de las obtenidas cuando se dejaba en superficie durante el otoño. Del mismo modo, a igualdad de manejo del rastrojo, el laboreo siguiendo las curvas de nivel redujo las pérdidas de suelo a un 42 y $62 \%$ de las que se registraron a favor de la máxima pendiente.

En consecuencia, el tratamiento que más erosión presentó fue en la parcela sembrada a favor de la pendiente y con enterrado tardío del rastrojo (diciembre), mientras que en la que se sembró por curvas de nivel y se enterró el rastrojo al finalizar el verano fue tan sólo el $21 \%$ de la registrada en el caso anterior.

Este manejo agronómico del suelo no sólo afectaba a la pérdida total de suelo, sino que las escorrentías también se veían condicionadas de forma similar. El manejo que se hacía del rastrojo cobraba más importancia cuando la dirección del laboreo era a favor de la pendiente, llegando a reducirse la escorrentía en un $50 \%$ a cuando se realizaba el enterrado tardío del rastrojo (diciembre), mientras que cuando la dirección del laboreo era perpendicular a la máxima pendiente, la escorrentía tan sólo representaba una reducción del $30 \%$ con respecto a enterrar el rastrojo en diciembre.

La influencia del manejo es de tal envergadura, que cuando se realizaba el enterrado temprano (octubre), la dirección del laboreo prácticamente no influía. Por tanto, la situación de los residuos (superficie o enterrado) durante las lluvias otoñales es un factor clave de cara a disminuir las escorrentías (fig. 4), superior incluso a la dirección del laboreo.

La conservación del agua del suelo es un objetivo importante en el manejo del suelo de cara a la producción vegetal. Este aspecto adquiere especial importancia en aquellas zonas semiáridas en donde los períodos de sequía son recurrentes y frecuentes. De aquí la importancia de establecer los mejores métodos de la labranza que permitan alcanzar este objetivo (Guzha, 2003) reduciendo las escorrentías.

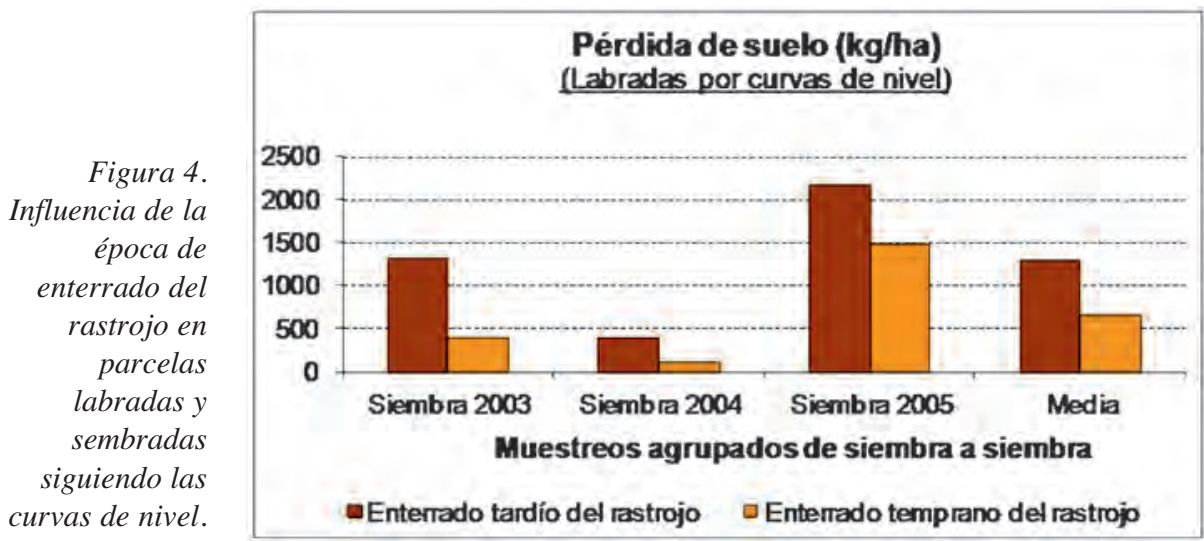




\subsection{Erosión en olivares}

Los cultivos leñosos manejados con laboreo en zonas en pendiente dan lugar a situaciones de riesgo elevado de erosión, hasta el punto de que sus pérdidas de suelo pueden suponer 4 veces las producidas bajo otros manejos (Fleskens y Stroosnijder, 2007). En este trabajo se estudió un olivar situado en una pendiente del $10 \%$ cerca de Aranjuez. Se registró una tormenta de $43 \mathrm{~mm}$, con picos de intensidad $\mathrm{I}_{10}$ de $55 \mathrm{~mm} \mathrm{~h}^{-1}$ (fig. 5) (Bienes et al., 2008), cuyo periodo de recurrencia es de 5 años (Elías Castillo y Ruiz Beltrán, 1979), y que se considera como un evento moderadamente erosivo, con una energía cinética calculada a partir de los diámetros de las gotas de agua de $627 \mathrm{~J} \mathrm{~m}^{-2}$ $\mathrm{mm}^{-1} \mathrm{y}$ un diámetro medio $\left(\mathrm{D}_{50}\right)$ de las gotas de lluvia de $2.92 \mathrm{~mm}$ (fig. 6) (Bienes et al., 2011). Las circunstancias previas al desencadenamiento de estas tormentas son importantes, y en este caso, la tormenta fue precedida 15 horas antes por una lluvia suave (25 $\mathrm{mm}$.), que saturaron en gran parte el suelo de agua.

Intensidad máxima en 10 minutos $\left(\mathrm{mm} \cdot \mathrm{h}^{-1}\right)$
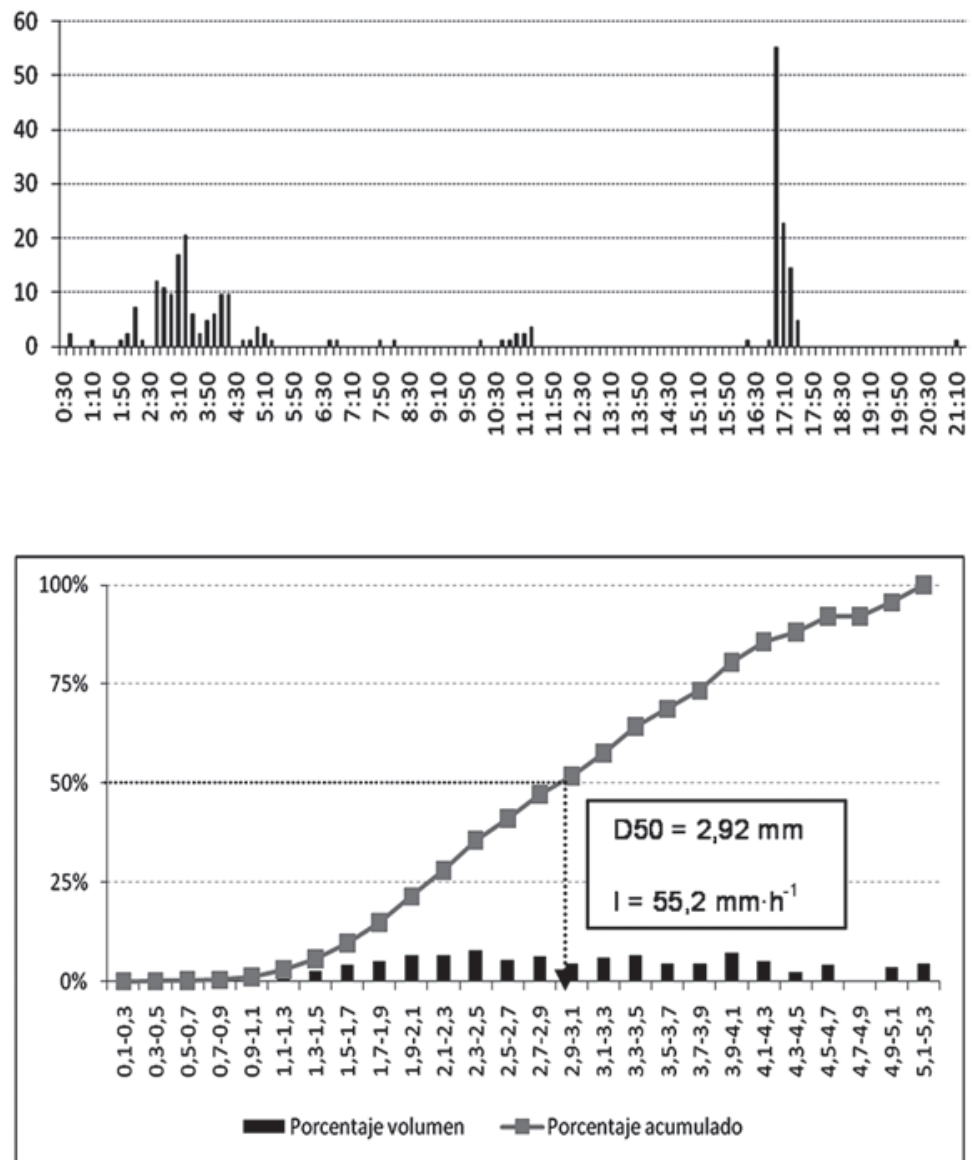

Figura 5.

Registro

pluviométrico

de la estación

meteorológica

automática

situada en la

zona de estudio.
Figura 6.

Distribución de los diámetros de las gotas de la tormenta y $D_{50}$. 


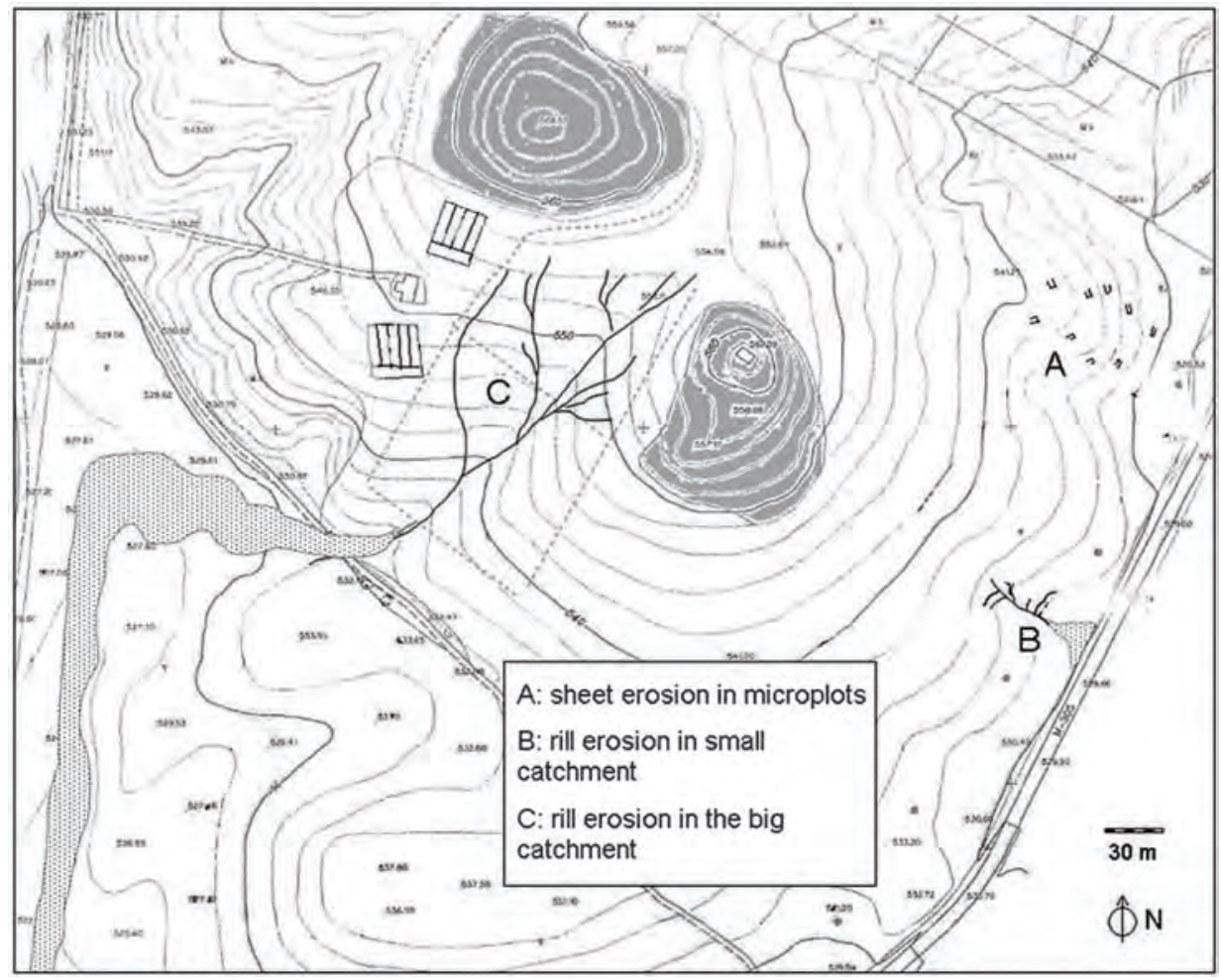

Figura 7. Situación de las 15 microparcelas (A) y de las dos minicuencas (B y C) con formación de regueros y zona de sedimentación (área punteada) correspondientes a los dos manejos del suelo según la dirección de laboreo. Minicuenca B: parcela labrada perpendicular a la pendiente. Minicuenca C: parcela labrada a favor de la pendiente.

La zona estudiada (fig. 7) abarcaba dos pequeñas cuencas (B y C) de 0.52 y 2.45 has de superficie, y próximo a ambas estaban instaladas 15 microparcelas de $1 \mathrm{~m}^{2}$ (A). Se estudiaron las pérdidas producidas por erosión en regueros y en las parcelas se estimó la erosión laminar o entre-regueros que tuvo lugar en ese mismo episodio.

Las pérdidas de suelo se estimaron en $7.60 \mathrm{Mg} \mathrm{ha}^{-1}$ en la minicuenca B y de 93.5 $\mathrm{Mg} \mathrm{ha}^{-1}$ en la minicuenca $\mathrm{C}$ (Bienes et al., 2008). La diferente magnitud de cada una de estas minicuencas incidió de forma notable en las pérdidas de suelo, formándose una gran red de surcos de dimensiones grandes en $\mathrm{C}$, mientras que en $\mathrm{B}$ los surcos no progresaron tanto al ser la longitud de la ladera mucho menor.

La erosión entre regueras medida en las microparcelas fue de $0.6 \mathrm{Mg} \mathrm{ha}^{-1}$. Se observa que un único episodio erosivo multiplica la pérdida de suelo tolerable en este entorno de suelos delgados y pobres en materia orgánica, que ronda la tonelada por hectárea y año (Vandekerckhove et al., 2000). No obstante, también es necesario destacar que no 
se deben hacer promedios anuales con la erosión producida en regueros, dado que se podría sobreestimar la pérdida, pues los episodios que los ocasionan no suelen tener frecuencia anual (Betts y De Rose, 1999).

\subsection{Erosión en viñedos}

Durante dos años (2008 y 2009) se estudiaron tres viñedos manejados con suelo desnudo mediante laboreo tradicional en las localidades de Belmonte de Tajo, Campo Real y Villaconejos (detalles en tabla 2). Se introdujeron también cubiertas vegetales en las calles de los viñedos para poder comparar las pérdidas de suelo bajo usos tradicionales y las pérdidas con manejos de cubiertas. Los tratamientos y características de este estudio se detallan en la tabla 9.

Tabla 9. Localidades de estudio. Ubicación y características de los viñedos y sus respectivos tratamientos.

\begin{tabular}{|c|c|c|c|}
\hline Localidades & Belmonte de Tajo & Campo Real & Villaconejos \\
\hline Altitud (m) & 750 & 820 & 608 \\
\hline Pendiente & $12 \%$ & $14 \%$ & $8 \%$ \\
\hline Edad del viñedo & 4 años & 7 años & 40 años \\
\hline Variedad de uva & Syrah & Tempranillo & Malvar \\
\hline Espaciado entre plantas $(\mathrm{m})^{*}$ & $3.5 \times 2.4$ & $2.9 \times 1.5$ & $2.9 \times 3.4$ \\
\hline Disposición vides & En vaso & En espaldera & En vaso \\
\hline \multicolumn{4}{|l|}{ Tratamientos: } \\
\hline T1 (control) & Laboreo & Laboreo & Laboreo \\
\hline $\begin{array}{l}\text { T2 (siembra solo año } 1) \\
\text { Dosis siembra }\left(\mathrm{kg} \mathrm{ha}^{-1}\right)\end{array}$ & $\begin{array}{l}\text { Brachypodium } \\
\text { distachyon } \\
40 \mathrm{~kg} \mathrm{ha}^{-1}\end{array}$ & $\begin{array}{l}\text { Brachypodium } \\
\text { distachyon } \\
40 \mathrm{~kg} \mathrm{ha}^{-1}\end{array}$ & $\begin{array}{l}\text { Brachypodium } \\
\text { distachyon } \\
40 \mathrm{~kg} \mathrm{ha}^{-1}\end{array}$ \\
\hline T3 (siembra y siega anuales) & $\begin{array}{l}\text { Vegetación } \\
\text { espontánea }\end{array}$ & $\begin{array}{c}\text { Secale cereale } \\
\text { (centeno) } 70 \mathrm{~kg} \mathrm{ha}^{-1}\end{array}$ & $\begin{array}{l}\text { Hordeum vulgare } \\
\text { (cebada) } 70 \mathrm{~kg} \mathrm{ha}^{-1}\end{array}$ \\
\hline Primera siembra & Invierno 2007 & Invierno 2006 & Invierno 2007 \\
\hline
\end{tabular}

* (distancia entre filas x distancia entre plantas de la misma fila)

En cada viñedo se instalaron 9 parcelas de $2 \mathrm{~m}^{2}$ ( 4 x $0.5 \mathrm{~m}$ ) (fig. 8), tres por cada tratamiento (fig. 8). Las cubiertas de centeno y de cebada se segaban y sembraban anualmente, mientras que las cubiertas de $B$. distachyon se sembraron únicamente el primer año de estudio, y nunca se segaron, por tanto se autosembraban anualmente. La cubierta de vegetación espontánea sí se segaba en primavera y aunque volvía a rebrotar, se dejaba evolucionar a partir del verano. 


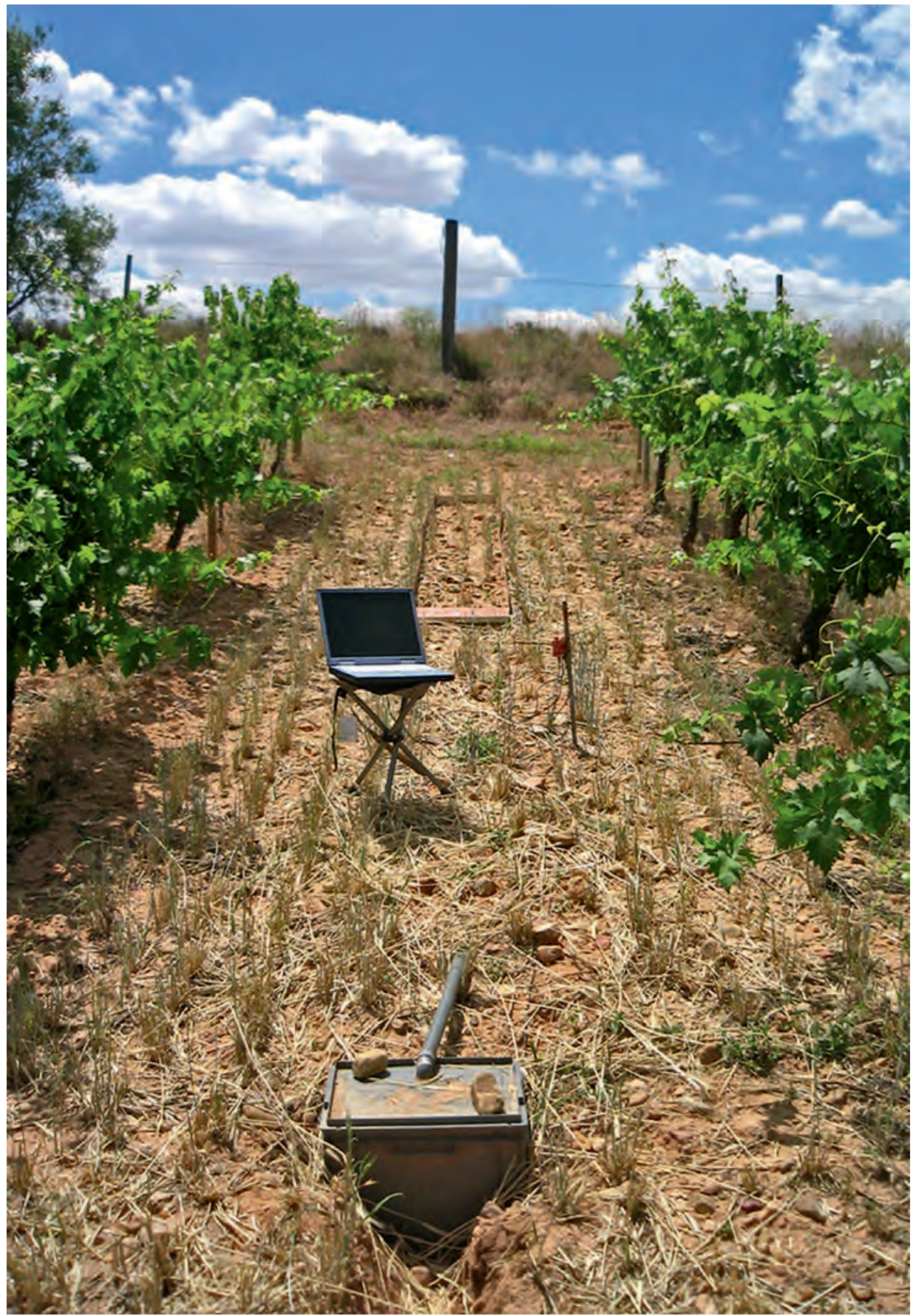

Figura 8. Detalle de calle de viñedo con parcela de $2 \mathrm{~m}^{2}$, en el centro de la calle donde se ha sembrado y segado centeno (Secale cereal). Campo Real, Madrid. 
Se obtuvieron por tanto, distintos grados de cobertura vegetal, 70-100\% en Brachypodium y entre $33-50 \%$ en el resto de tratamientos. El laboreo mantuvo coberturas por debajo del $3 \%$ a lo largo de todo el año.

La pérdida media de suelo fue de $35 \mathrm{~g} \cdot \mathrm{m}^{-2}$ en Belmonte de Tajo y Campo Real, y de $178 \mathrm{~g} \cdot \mathrm{m}^{-2}$ en Villaconejos (tabla 10). Las cubiertas redujeron estos valores de erosión obtenidos con el laboreo tradicional. En el caso de las cubiertas permanentes más de un $50 \%$, y fue particularmente efectiva en Campo Real, donde el tratamiento llevaba un año más de experimentación (Marqués et al., 2010). La vegetación espontánea redujo un $34 \%$, el centeno un $57 \%$ y la cebada un $82 \%$. Es de destacar que estas dos últimas cubiertas no tuvieron efectos en la producción de uva, cosa que sí se produjo con las otras cubiertas vegetales ensayadas debido a la competencia por el agua (Marqués et al., 2009).

Tabla 10. Pérdida anual de suelo (media \pm desviación estándar) en el periodo 2008-2009. Parcelas de $2 \mathrm{~m}^{2}$ (4 longitud $x 0.5$ anchura).

\begin{tabular}{|l|c|}
\hline Localidad & $\begin{array}{c}\text { Pérdida Suelo } \\
\left(\mathbf{g} \cdot \mathbf{m}^{-2}\right)\end{array}$ \\
\hline Belmonte de Tajo \\
\hline Laboreo & $35.1 \pm 16.9$ \\
\hline Brachypodium & $16.5 \pm 14.3$ \\
\hline V.E. & $23.7 \pm 13.7$ \\
\hline Campo Real & $35.3 \pm 7.7$ \\
\hline Laboreo & $2.4 \pm 1.1$ \\
\hline Brachypodium & $15.25 \pm 5.0$ \\
\hline Centeno & $178.6 \pm 43.1$ \\
\hline Villaconejos & $27.6 \pm 22.8$ \\
\hline Laboreo & $31.8 \pm 10.0$ \\
\hline Brachypodium
\end{tabular}

Si pudiéramos expresar estas cifras en toneladas por hectárea, salvando las dificultades de los cambios de escala (Pierson et al., 1994), la pérdida media anual en este periodo, caracterizado por su baja erosividad en las lluvias, habría sido entre 0.4 y 1.8 $( \pm 0.23) \mathrm{t} \mathrm{ha}^{-1} \mathrm{año}^{-1}$ en viñedos con laboreo tradicional; entre 0.02 y $0.3( \pm 0.13) \mathrm{t} \mathrm{ha}^{-1}$ año ${ }^{-1}$ en viñedos con cubiertas de Brachypodium distachyon; de $0.24( \pm 0.14) \mathrm{t} \mathrm{ha}^{-1} \mathrm{año}^{-1}$ en viñedos con vegetación espontánea en las calles; $0.15( \pm 0.05) \mathrm{t} \mathrm{ha}^{-1} \mathrm{año}^{-1}$ con centeno en las calles y $0.32( \pm 0.10) \mathrm{t} \mathrm{ha}^{-1} \mathrm{año}^{-1}$ con cebada en las calles del viñedo. Shinjo et al., (2000), también han publicado pérdidas de suelo semejantes en este mismo entorno climático semiárido, oscilando entre las $0.4 \mathrm{t} \mathrm{ha}^{-1} \mathrm{año}^{-1}$ en suelos con cubiertas vegetales y $1.4 \mathrm{t} \mathrm{ha}^{-1} \mathrm{año}^{-1}$ en suelos manejados con laboreo tradicional. 
También es importante destacar la magnitud del coeficiente de escorrentía (tabla 11). El laboreo tradicional produce siempre más escorrentía que los otros tratamientos, esto demuestra que uno de los motivos por los cuales se realiza el laboreo, que pretende aumentar la infiltración, no funciona a escala anual, aunque sí se produce mayor infiltración inmediatamente después de realizado el laboreo.

Tabla 11. Coeficiente de escorrentía encontrado en el periodo 2008-2009 en los viñedos estudiados. Parcelas de $2 \mathrm{~m}^{2}$.

\begin{tabular}{|l|c|c|c|c|}
\hline \multirow{2}{*}{ Localidad } & \multirow{2}{*}{$\mathbf{n}$} & \multicolumn{3}{|c|}{ Coeficiente de escorrentía } \\
\cline { 3 - 5 } & & Laboreo & Brachypodium & Tercer tratamiento \\
\hline Belmonte & 141 & $0.44 \%$ & $0.30 \%$ & V.E.: $0.34 \%$ \\
\hline Campo Real & 111 & $2.07 \%$ & $0.61 \%$ & Centeno: $1.10 \%$ \\
\hline Villaconejos & 121 & $11.37 \%$ & $1.76 \%$ & Cebada: $2.65 \%$ \\
\hline
\end{tabular}

En el otoño de 2008 se realizaron ensayos de lluvia simulada en Campo Real sobre estas parcelas de $2 \mathrm{~m}^{2}$ para analizar de forma más detallada la escorrentía y la pérdida de suelo en dos momentos diferentes del ciclo anual: después de haber transcurrido varios meses desde el laboreo de primavera e inmediatamente después de realizar la siembra del centeno, la cual fue precedida de una labor preparatoria del terreno; el tratamiento de Brachypodium no estaba sujeto a cambios. Los resultados se muestran en la tabla 12. La pérdida de suelo se duplica como consecuencia de las labores realizadas para la siembra en el centeno, permanece igual en el tratamiento de Brachypodium y casi se cuadruplica en el caso del laboreo. Los valores correspondientes a $\mathrm{g} \cdot \mathrm{m}^{-2}$ resultan de calcular los sedimentos generados por cada lluvia (tabla 10) en función de su coeficiente de escorrentía. La erosión producida por esta única lluvia sería inferior a los $0.5 \mathrm{~g} \cdot \mathrm{m}^{-2}$ en todos los tratamientos con cubierta, antes o después del manejo necesario, pero es de $3.9 \mathrm{~g} \cdot \mathrm{m}^{-2}$ en el caso del suelo desnudo sin labrar, y de $14.6 \mathrm{~g} \cdot \mathrm{m}^{-2}$ en el suelo recién labrado.

Tabla 12. Erosión y escorrentía: la primera lluvia se realizó con los suelos tal y como se encontraban inmediatamente antes de las labores otoñales, la segunda lluvia se realizó inmediatamente después de sembrar el centeno y labrar el tratamiento tradicional. Letras distintas indican diferencias significativas (para $p<0.01$ ).

\begin{tabular}{|l|c|c|c|}
\hline \multirow{2}{*}{ Variables } & \multicolumn{3}{|c|}{ Tratamientos } \\
\cline { 2 - 4 } & Centeno & Brachypodium & Laboreo \\
\hline Lluvia simulada previa & & & \\
\hline Sedimento generado $\left(\mathrm{g} \cdot \mathrm{l}^{-1}\right)$ & $0.27 \pm 0.31 \mathrm{a}$ & $0.42 \pm 0.84 \mathrm{a}$ & $0.93 \pm 1.03 \mathrm{~b}$ \\
\hline Coeficiente de escorrentía $(\%)$ & $4.4 \pm 6 \mathrm{a}$ & $3.7 \pm 8 \mathrm{~b}$ & $26 \pm 35 \mathrm{c}$ \\
\hline Lluvia simulada posterior a & Siembra de centeno & Inalterado & Recién labrado \\
\hline Sedimento generado $\left(\mathrm{g} \cdot \mathrm{l}^{-1}\right)$ & $0.60 \pm 1.32 \mathrm{a}$ & $0.47 \pm 0.99 \mathrm{~b}$ & $3.46 \pm 1.98 \mathrm{c}$ \\
\hline Coeficiente de escorrentía $(\%)$ & $4 \pm 5 \mathrm{a}$ & $4 \pm 12 \mathrm{~b}$ & $31 \pm 36 \mathrm{c}$ \\
\hline
\end{tabular}


Los eventos extremos multiplican la pérdida de suelo, tal como se ha podido comprobar en el evento erosivo que tuvo lugar el 20 de mayo de 2007, que con una duración de 4 horas acumuló 43 mm de lluvia. Según Elías Castillo y Ruíz-Beltrán (1979), este tipo de eventos tiene lugar con una periodicidad inferior a los 5 años (tabla 1). En los 10 minutos de mayor intensidad, las parcelas con laboreo tradicional mostraron coeficientes de escorrentía del $40 \%$ y una pérdida de suelo media de $787 \mathrm{~g} \mathrm{~m}^{-2}$ (Marqués et al., 2009).

\section{Conclusiones}

A lo largo del periodo de estudio (1994-2009), en diferentes localidades, con diferentes métodos y manejos se ha podido comprobar que las pérdidas de suelo en zonas agrícolas dedicados a cereales, pastizal, girasol, olivares o viñedos son generalmente inferiores al límite de $17 \mathrm{t} \mathrm{ha}^{-1} \mathrm{año}^{-1}$ recomendado por Pimentel et al. (1995), pero están en el orden de los valores expresados por Verheijen et al. (2009), entre 0.3 y $1.4 \mathrm{t} \mathrm{ha}^{-1}$ año-1 que sí pueden afectar la conservación de suelos vulnerables. Sin embargo, con el suelo desnudo (por laboreo o mediante empleo de herbicidas), las pérdidas de suelo se incrementan notablemente alcanzando valores medios anuales de $20 \mathrm{t} \mathrm{ha}^{-1} \mathrm{año}^{-1}$. En condiciones de baja erosividad, el suelo desnudo sufre una pérdida media de suelo de $1.3 \mathrm{t} \mathrm{ha}^{-1} \mathrm{año}^{-1}$. Este valor puede verse incrementado en varios órdenes de magnitud con eventos erosivos. Los eventos aquí mencionados, dieron lugar a pérdidas de $93.5 \mathrm{t} \mathrm{ha}^{-1}$ en olivares y de $7.8 \mathrm{tha}^{-1}$ en viñedos durante un único evento.

La vegetación espontánea usada como protección del suelo limita la erosión, arrojando cifras siempre inferiores a los $1.76 \mathrm{t} \mathrm{ha}^{-1} \mathrm{año}^{-1}$, valor que podría considerarse en esta zona como pérdida de suelo máxima admisible.

En el caso de las cubiertas de gramíneas segadas y sembradas, se obtienen pérdidas entre 0.15 y $0.32 \mathrm{t} \mathrm{ha}^{-1} \mathrm{año}^{-1}$.

La utilización de cubiertas en las calles del viñedo y olivar se ha mostrado como una herramienta muy eficaz para disminuir la pérdida de suelo, si bien las recomendaciones que puedan hacerse respecto a la especie elegida y su manejo estarán en función de las características de la zona.

\section{Agradecimientos}

Al Ministerio de Ciencia y Tecnología, al INIA y a la Comunidad de Madrid, por la financiación de los proyectos SC93-022-C2, SC98-031-C3, 07M/0009/2001, FP02-DR1, 07M/0011/2002, FP04-DR1, FP06-DR3VID, FP07-AG1 y RTA2007-00086-00-00.

\section{Referencias bibliográficas}

Arshad, M. A., GiLl, K. S. (1997). Barley, canola and wheat production under different tillage-fallow-green manure combinations on a clay soil in a cold, semiarid climate. Soil \& Tillage Research, 43: 263-275. 
Basic, F., Kisic, I., Mesic, M., Nestroy, O., Butorac, A. (2004). Tillage and crop management effects on soil erosion in central Croatia. Soil \& Tillage Research, 78 : 197-206.

BAzzoffi, P. (2009). Soil erosion tolerance and water runoff control: minimum environmental standards. Reg. Environ Change, 9 (3): 169-179.

Betts, H. D., De Rose, R. C. (1999). Digital elevation models as a tool for monitoring and measuring gully erosion. Int. J. Appl. Earth Obs. \& Geoinf., 1: 91-101.

Bienes, R., Torcal SÁinz, L. (1997). Influencia del manejo del suelo sobre erosión en depósitos de terraza (El Encín y Marchamalo). Cuaternario y Geomorfología, 11: 113-124.

Bienes, R. Guerrero-Campo, J., Aroca, J. A., Gómez, B., Nicolau, J. M., Espigares, T. (2001). Evolución del coeficiente de escorrentía en campos agrícolas del centro de España con diferentes usos del suelo. Ecología, 15: 23-36.

Bienes, R., MArqués, M. J. (2002). Pérdida de nutrientes de suelos con distintos tipos de usos del territorio en el centro de la Península Ibérica. En: Actas del Congreso Nacional de Medio Ambiente, CONAMA, pp. 1-17, Madrid.

Bienes, R., Moré, A., Marqués, M. J., Moreiro, S., Nicolau, J. M. (2005). Efficiency of different plant cover to control water erosion in central Spain. En: Sustainable Use and Management of Soils. Arid and Semiarid Regions. Adv. Geoecol., 36: 155-162.

BIENES, R., MARQUES, M. J. (2008). Rill and interril erosion produced by a single-storm event in an olive grove in central Spain. Proceedings of EUROSOIL 2008, Control No. 2008-A-804, Vienna (Austria). August 25-29, 2008.

Bienes, R., Ruíz-Colmenero, R., Arévalo, D., Marqués Pérez, M. J. (2011). Distribución del tamaño de gota de una lluvia natural y su energía cinética. En: Control de la degradación y uso sostenible del suelo (Ortiz Silla, R. y Sánchez Navarro, A. Eds.), pp. 165-168, Murcia.

Boardman, J., Poesen, J., Evans, R. (2003). Socio-economic factors in soil erosion and conservation. Environmental Science \& Policy, 6: 1-6

Calatrava, J., Barberá, G. G., Castillo, V. M. (2011). Farming practices and policy measures for agricultural soil conservation in semi-arid Mediterranean areas: the case of the Guadalentín basin in southeast Spain. Land Degrad. Develop., 22: 58-69.

Cerdá, A. (1999). Simuladores de lluvia y su aplicación a la Geomorfología. El estado de la cuestión. Cuadernos de Investigación Geográfica, 25: 45-84.

Christiansen, J. E. (1942). Irrigation by sprinkling. University of California Agric. Exp. Sta. Bulletin, Bulletin 670- Experiment Station, 124 pp. California, US.

Coutinho, M. A., Tomás, P. P. (1995). Characterization of raindrop size distributions at the Vale Formoso Experimental Erosion Center. Catena, 25: 187-197. 
Dunjó, G., PARdini, G., GisPert, M. (2004). The role of land use-land cover on runoff generation and sediment yield at a microplot scale, in a small Mediterranean catchment. Journal Arid Environment, 57 (2): 239-256.

EC (European Commission) (2006). A strategy to keep Europe's soils robust and healthy. http://ec.europa.eu/environment/soil/index.htm.

European Enviroment Agency. European Topic Centre on Land use and sPatial INFORMATION (2010). The European Enviroment: State and Outlook 2010. Land Use. EEA, Copenhagen, 2010. Luxembourg: Publications Office of the European Union, 2010. $52 \mathrm{pp}$.

Elías Castillo, F., Ruiz Beltrán, L. (1979). Precipitaciones máximas en España. Estimaciones basadas en métodos estadísticos. ICONA-Monografías, vol. 21.

Fleskens, L., Stroosnidder, L. (2007). Is soil erosion in olive groves as bad as often claimed? Geoderma, 141: 260-271.

García-Ruiz, J. M. (2010). The effects of land uses on soil erosion in Spain: A review. Catena, 81: 1-11.

García-Ruiz, J. M., Lana-Renault, N. (2011). Hydrological and erosive consequences of farmland abandonment in Europe, with special reference to the Mediterranean region-A review. Agric. Ecosyst. Environ., doi:10.1016/j.agee.2011.01.003.

González-Hidalgo, J. C., de luís Arrillaga, M., Peña Monné, J. L. (2005). Los eventos extremos de precipitación, la variabilidad del clima y la erosión del suelo. Reflexiones ante el cambio del clima en los sistemas mediterráneos. Rev. C. y C., 19 (1-2): 49-62.

GuzHA, A. C. (2003). Effects of tillage on soil microrelief, surface depression storage and soil water storage. Soil and Tillage Research, 76 (2): 105-114.

LÓPEZ-BERMúdez, F. (2002). Erosión y desertificación. Nivola Ed., Madrid (Spain).

Marqués, M. J., Jiménez, L., Pérez-Rodríguez, R., García-Ormaechea, S., Bienes, R. (2005). Reducing water erosion in a gypsic soil by combined use of organic amendent and shrub revegetation. Land Degrad. Develop., 16: 339-350.

Marqués, M. J., Bienes, R., Jiménez L., PÉrez-Rodríguez, R. (2007). Effect of vegetal cover on runoff and soil erosion under light intensity events. Rainfall simulation over USLE plots. Sci. Total Environ., 378: 161-165.

Marqués, M. J., Bienes, R., Pérez-RodríGuez, R., Jiménez, L. (2008). Soil degradation in Central Spain due to sheet water erosion by low-intensity rainfall events. Earth Surface Processes and Landforms, 33: 414-423.

Marqués, M. J., Ruiz-Colmenero, R., Garcia-Muñoz, S., Cabello, F., MuñozOrganero, G., Pérez-Jiménez, M. A., Bienes, R. (2009). Protección contra la erosión versus productividad en viñedos. Ensayos de cubiertas vegetales en cultivos en pendiente. En: Advances in Studies on Desertification (Romero, A., Belmonte, F., Alonso, F., López-Bermúdez, F., eds.), pp. 319-322, Murcia. 
Marqués M. J., Velasco, A., Ruiz-Colmenero, M., Cuadrado, J., Bienes, R. (2010). To what extent are farmers willing to collaborate to control land degradation? En: Land Degradation Processes and Assessment: wind erosion, interrill erosion, gully erosion, land cover features (Vermang, J., Gabriels, D., Cornelis, W. M., De Boever, M., eds.) Unesco Chair of Eremology, Ghent University, Belgium, 173 pp.

Marqués, M. J., García-Muñoz, S., Muñoz-Organero, G., Bienes, R. (2010). Soil conservation beneath grass cover in hillside vineyards under Mediterranean Climatic conditions (Madrid, Spain). Land Degrad. Develop., 21 (2): 122-131.

Martínez-Mena, M. A., Abadia, R., Castillo, V., Albaladejo, J. (2001). Diseño experimental mediante lluvia simulada para el estudio de los cambios en la erosión del suelo durante la tormenta. Cuaternario y Geomorfología, 15 (1-2): 31-43.

Nicolau, J. M., Bienes, R., Guerrero-Campo, J., Aroca, J. A., Gómez, B., Espigares, T. (2002). Runoff coefficient and soil erosion rates in croplands in a Mediterranean-continental region, in Central Spain. En: Man and Soil at the Third Millenium (Rubio J. L., Morgan, R. P. C., Asíns, S., Andreu, V., eds.) Geoforma Ediciones, pp. 1359-1368, Logroño.

Pierson, F. B., Blackburn, W. H., Van Vactor, S. S., Wood, J. C. (1994). Partitioning small scale spatial variability of runoff and erosion on sagebush rangeland. Water Resources Bulletin, 30: 1081-1089.

Pimentel, D. (2006). Soil erosion: a food and environmental threat. Environment, Development and Sustainability, 8: 119-137.

Pimentel, D., Harvey, C., Resosudarmo, P., Sinclair, K., Kurz, D., McNair, M., Crist, S., ShPritz, L., FitTon, L., SAFFouri, R., Blair, R. (1995). Environmental and economic cost of soil erosion and conservation benefits. Science, 267: 1117-1123.

Rainbault, B. A., Vyn, T. J., TollenaAr, M. (1990). Corn response to rye cover crop management and spring tillage systems. Agronomy Journal, 82 (6): 1088-1093.

Ramos, M. C. (2006). Soil water content and yield variability in vineyards of Mediterranean northeastern Spain affected by mechanization and climate variability. Hydrol. Process., 20: 2271-2283.

Renschler, C. S., Harbor, J. (2002). Soil erosion assessment tools from point to regional scales-the role of geomorphologists in land management research and implementation. Geomorphology, 47: 189-209.

Romero Díaz, A., López-Bermúdez, F., Belmonte-Serrato, F. (1998). Erosión y escorrentía en el campo experimental de "El Ardal" (Murcia). Nueve años de experiencias. Papeles de Geografía, 27: 115-130.

Romero DíAz, A. (2003). Influencia de la litología en las consecuencias del abandono de tierras de cultivo en medios mediterráneos semiáridos. Papeles de Geografía, 38: 151-165. 
Ruiz-Colmenero, M., Bienes, R., Marques, M. J. (2010). Runoff and soil loss under different land management practices in vineyards: grass cover treatments and traditional tillage. Results from simulated rainfall. Geophysical Research Abstracts, Vol. 12, EGU2010-4153-6, EGU General Assembly 2010.

Shinjo, H., Fujita, H., Gintzbuger, G., Kosaki, T. (2000). Impact of grazing and tillage on water erosion in northeastern Syria. Soil Sci. Plant Nutr., 46: 151-162.

Simoncini, R. (2009). Developing an integrated approach to enhance the delivering of environmental goods and services by agro-ecosystems. Reg Environ Change, 9: 153-167.

Souchère, V., King, C., Dubreui, N., Lecomte-Morel, V., Le Bissonnais, Y., ChaLAT, M. (2003). Grassland and crop trends: role of the European Union Common Agricultural Policy and consequences for runoff and soil erosion. Environmental Science \& Policy, 6: 7-16.

Sturgul, S. J., Daniel, T. C., Mueller, D. H. (1990). Tillage and canopy cover effects on interrill erosion from first-year alfalfa. Soil Science Society of America Journal (USA), 54 (6): 1733-1739.

Vandekerckhove, L., Poesen, J., Oostwoud-Wijdenes, D., Gyssels, G., Beuselinck, L., DE LunA, E. (2000). Characteristics and controlling factors of bank gullies in two semi-arid mediterranean environments. Geomorphology, 33: 37-58.

Verheijen, F. G. A., Jones, R. J. A., Rickson, R. J., Smith, C. J. (2009). Tolerable versus actual soil erosion rates in Europe. Earth-Sci. Rev., 94: 23-38.

WischmeIer, W. H., Smith, D. D. (1978). Predicting Rainfall Erosion Losses. A Guide to Conservation Planning, USDA Agric, U.S. Govt. Printing Office, Washington, US. Handbook, no 537,58 pp.

ZANCHI, C., Torri, D. (1980). Evaluation of rainfall energy in central Italy. En: Assessment of erosion (De Boodt, J., Gabriels, D., Ed.) Wiley, pp. 133-142, London, UK. 